\title{
The Art of Ancient Caps From Small River Cemetery, Xinjiang, China
}

\author{
Xiaoyu Xin ${ }^{1, * *}$ Rulin Yang ${ }^{2}$ Xiaojing Kang ${ }^{3}$
}

${ }^{1}$ College of Textile and Clothing, Xinjiang University, Urumqi, Xinjiang, China

${ }^{2}$ China National Silk Museum, Hangzhou, Zhejiang, China

${ }^{3}$ Xinjiang Archeology Institute, Urumqi, Xinjiang, China

${ }^{* *}$ Corresponding author. Email: xinxiaoyu00@163.com

\begin{abstract}
Based on the archaeological evidence, the paper analyzed the art and craft of the ancient caps unearthed from Small River Cemetery, Xinjiang, China, which has a history of 3500-4000 years. From the detailed description of style, material, size, color, chin-cords and ornaments of those caps, the article unveils the headgear culture of the Bronze Age people of Lop-Nor region, and shows us a strong primitive aesthetics of these ancient people. Meanwhile, the discussion at end of the paper made some comparisons on the antique caps excavated from other Bronze Age tombs in Tarim basin as well as some other ancient sites outside China.
\end{abstract}

\section{Keywords: felt caps, Small River Cemetery, Xinjiang, Bronze Age, headgear}

\section{INTRODUCTION}

The felt caps are important category of costume unearthed from Small River Cemetery (SRC, Chinese: Xiaohe Cemetery). As the earliest pioneer, Vivi Sylwan had done lots of studies about the nine felt caps found by Folke Bergman from the SRC5 during the 1930s1940s [1]. After the excavation directed by Xinjiang Institute of Archaeology (XIA) during 2002-2005, a quantity of new archaeological materials had been found in this mysterious cemetery, and the felt caps are one of them.

There were more than fifteen felt caps exhumed from the tombs, let alone those found near the cemetery. The frequency of felt caps appeared in the graves attested that they were of great importance to people who buried here 4000-3500 years ago. Thanks to the dry climate and salty soil of Tarim Basin, most of the felt caps from SRC were well persevered. In the following passages, we focus on the newfound felt caps from this important archeological site of Bronze Age Xinjiang, China, and try to give a quick glimpse to the features of those ancient headgears.

\section{STYLE}

The felt caps from SRC are of very simple style. Most of them are round caps, and feathers and strings were used as decoration on the surface of the caps. Sometimes there are also weasel pelts hanging in front

*Fund: The paper is funded by Scientific Research Program of the Higher Education Institution of XinJiang. (Grant NO.XJEDU2018SY013) of the caps. According to the different shapes of the hats, we can divide them into 2 main types, straightedge caps and hood-like caps.

Most of the felt caps from SRC are straight-edge caps without ear-flaps. The bottom edges of this type are straight and in the same horizontal line. There are also 2 different styles of the crown shapes of these straight-edge caps. Style A is with slightly pointed crown while style B with round crown. And it seems the pointed-crown style were more popular in this ancient group of people. Both of these two styles of caps had been discovered by Bergman before, and there are more evidence found after the new excavation of the cemetery. (See "Fig. 1")

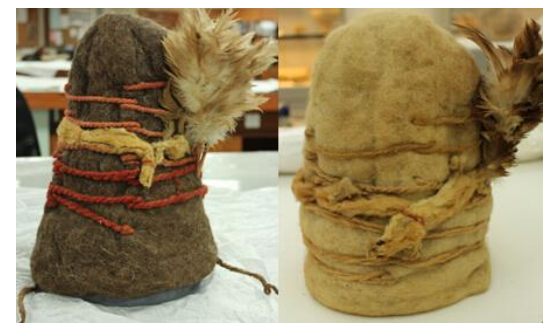

Fig. 1. Straight-edge felt caps, pointed and round crown.

The other type of the felt caps from SRC is the hood-like ones. Unlike the straight edge cap, there is a pair of crescent ear-flaps extended from the bottom edge of the cap. These hood-like caps are very rare comparing with those without ear-flaps, and we only found 2 caps belonged to this type. It should be pointed out that both of them were found at the northern part of the cemetery, which is known with the same culture 
characteristics with the fourth and fifth layers of the southern part of SRC. That means it is possible that people who wearing the felt caps with ear-flaps here has an older historical background than those wearing straight-edge caps, but more evidence are still needed to prove that. (See "Fig. 2")

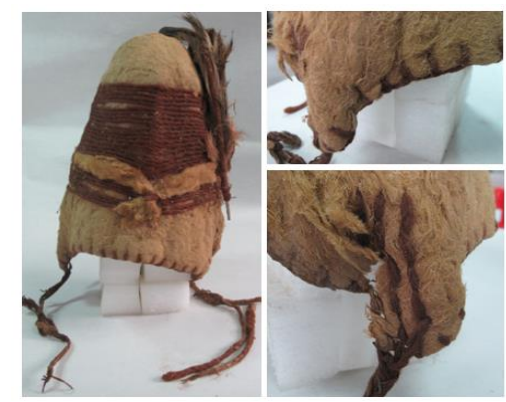

Fig. 2. Hood-like felt caps.

\section{MATERIALS AND TECHNIQUES}

As known, the headgear from SRC is mainly made of felt, which is made through a process of rolling and pressing wool under a condition of humidification and heating. The felt caps from SRC are one of the earliest evidences for felt using in Tarim Basin. Most of the felt caps from SRC are coarse and porous, uneven in thickness. The thickness of the felts was varying from 2.1-10mm. Even at different parts of the same cap, the data could differ a lot. It seems they are not with good quality compares with the following finds from other place in Xinjiang. As for the process of hatting, since there is no seam stitches on the surface of the caps, we presume that the ancient hat makers might first felting wool into a piece of flattened sheet, then put the wool sheet on a hat-block, probably made of wood, and felting the edges together to make a finished cap. Unfortunately, the evidence about hatting process is too scanty to confirm this hypothesis.

The felting techniques were originated from the fiber processing skills used by nomadic tribes in ancient Central Asia, which can be dated back to 8000 years ago [2]. Barber noted that the first well-documented specimen of felt was found in an early bronze II sanctuary in Anatolia [3]. She also implied that the wool techniques in Tarim Basin were originated from the Ancient Near East, where the woolly sheep developed from the nonwoolly ones around 4000BC [4]. Meanwhile, some documents from Chinese historical records suggest that the nomadic tribes who lived in the north part of China before Han dynasty had already mastered the techniques of felting [5]. They use felt to make headgear, footwear, garment and tents. However, the evidence about how the felting technique was utilized by the SR (Small River) people is still lack, and all the possible links between east and west during Bronze and Early Iron Age are still need to be proved.
It is interesting to note that felting still play a very important role in today's daily life of Xinjiang ethnic minorities such as Uighur, Kazak and Khalkhas. Further research is still needed to attest whether there is any connections between the felt works of the Bronze Age SR people and today's felt making in Xinjiang.

\section{SIZE}

The sizes of the felt caps from SRC are different from each other. There are two main measurements of the caps, one is the height, and the other one is the inside width. The height refers to the vertical height from the top to the bottom edge of the caps while the inside width refers to the circumference of the inside edge. Basically, from these two measurements, we always can find a foundation to compare the size of those felt caps. The heights of the caps from SRC are varying from $16-35 \mathrm{~cm}$. And the inside width of those caps is from $50-83 \mathrm{~cm}$.According to all the felt caps we have measured, the largest one is the cap 03XHM13:9 (H.32 cm. IW (Inside Width). $83 \mathrm{~cm}$ ) while the smallest one is the cap TEMP $14(\mathrm{H} .16 \mathrm{~cm}$. IW. $50 \mathrm{~cm})$, which is a cap for child. (See "Fig. 3")

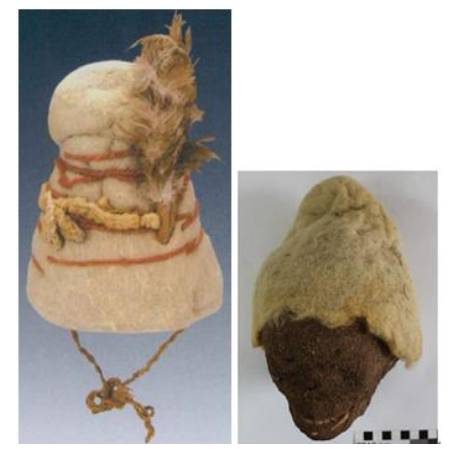

Fig. 3. Biggest and the smallest caps.

\section{COLOR}

Just like the cloaks and loincloths, the colors of the felt caps from SRC usually stay undyed. The nature color of wool can be easily seen among the textiles unearthed from the cemetery. Those colors include the felt white, beige, natural tan, natural brown, dark brown, etc. Apart from the nature wool color, the red and yellow dyestuff can be found in the strings trimming on the surface of the felt caps. Red is one of the favorite color which were used widely in the decoration of the textiles from SRC. This is also evident by the red stripes woven on the woolen cloaks and printed on the leather boots. Actually, it is hard to tell what the exact purpose of this red dye used here. Is that just for ornamental reason or some primitive religious purpose? The answer is still unclear. (See "Fig. 4") 


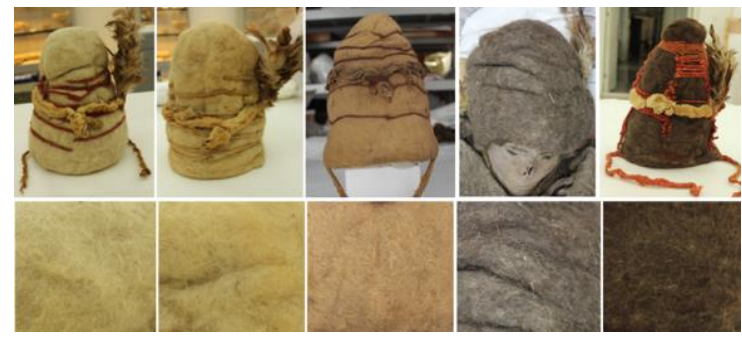

Fig. 4. Natural wool color of felt caps.

One thing should be noted is that almost all the string trimmings were with different color against the felt background. Most of the strings are different shades of red against dark-brown or yellowish white felt, providing a strong visual impact. The only exception is cap 03XHM28:11. It has the beige strings against the yellowish white felt. The color of the threads is not obvious at all. It is hard to say whether the strings had been dyed and lost their color or they had just keep undyed for thousands of years. Another cap 04XHM59: 9 may give some clue. This is a somewhat damaged felt cap with the threads of red color faded and spread to the vicinity. That means how to fix the red dye and keep threads colorfast was still a problem for SR natives. It is possible that the color of strings found in SRC were dyed by some nature dyes, such as madder and weld, and the dyestuff was presumably extracted from plants like rubia tinctorum and reseda lutea. The raw material of the nature dye was so limited that they were just used to dye a small quantity of threads instead of large quantity of wool. That may explain why the fabrics from SRC were all keep a nature color of wool, and only a few strings were dyed into red and yellow. It is no doubt that this red color has some significance in those people's daily life. (See "Fig. 5")

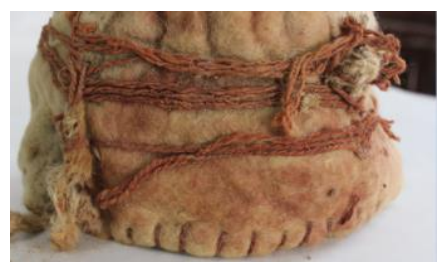

Fig. 5. The faded red color.

\section{CHIN-CORDS}

As for the methods of application, the chin-cords cannot be ignored. There are two chin cords for each felt cap from SRC, and they are always stitched to the edge by the two sides of the caps so that the caps can be fastened by tying the cords under the chin. From the physical remains of those chin-cords, two different ways can be recognized to deal with the threads after stitch them to the caps. One is twisting, and the other is plaiting.
The twisting way to make a chin-cord is as follows: First, make the yarn go through the felt. Second, stitch the yarn onto the edge of the cap. Third, fasten the two ends of the string and twist them first s-wise then $\mathrm{z}$ wise, and finally make it into a cord. (See "Fig. 6")

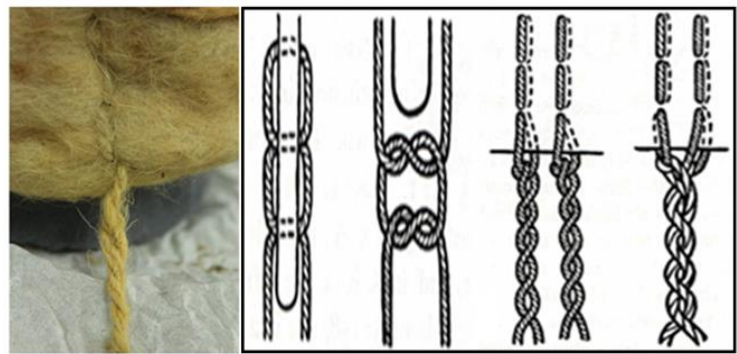

Fig. 6. Twisted chin-cord

Another way to make a chin-cord is plaiting. And the process of plaiting chin-cord consists of following steps: First, stitch several rows (3-4) of the yarns onto the side edge of the cap, and then plait the extending yarns together into a braid. There are also lots of examples for this kind of chin-cords, especially in the northern part of the cemetery. (See "Fig. 7")

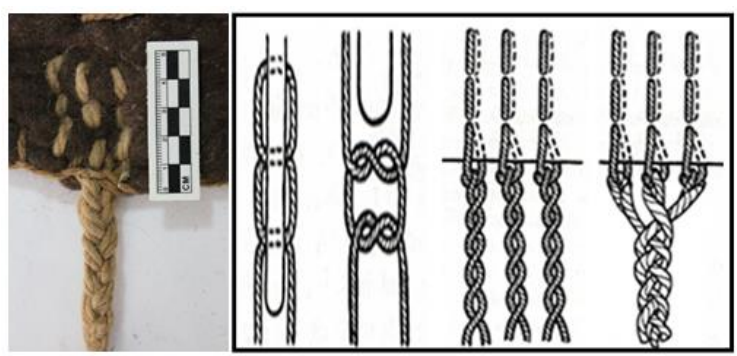

Fig. 7. Plaited chin-cord [6]

\section{ORNAMENTS}

Apart from the basic felt, people from the Bronze Age SRC also used some common ornaments to decorate the caps. Based on the different materials, those ornaments can be mainly divided into 3 categories: strings, feathers, and Weasel pelts.

\section{A. Strings}

One of the most characteristic ornaments of the felt caps from SRC is the string trimmings, which also can be called yarns or threads ornaments. Most of these threads are z-spun and s-twined, with a thickness about $0.2-0.5 \mathrm{~cm}$. Sometimes they were dyed into red or yellow, and sometimes just kept the nature look. It is interesting to note how the ancient SR natives use various threads to decorate their lives. Evidence that Strings served as necklace, bracelet and headgear ornaments can be found everywhere in this graveyard. 
As for the felt caps, the contrast of colored threads against the beige or brown felt give a strong visual effect.

The string trimmings were usually stitched on the surface of the cap, and there are two types of those stitches, stem-stitches and blanket-stitches.

Stem-stitches were always used on the body part of the caps .They begin a short distance from the edge of the cap. The threads run spirally and are stitched 4 times in one round: at front, at the back, and at both two sides. And the strings between those fastenings are free. The thread could start from either bottom or the top part of the cap, and then go spiral to another area. (See "Fig. $8 ")$

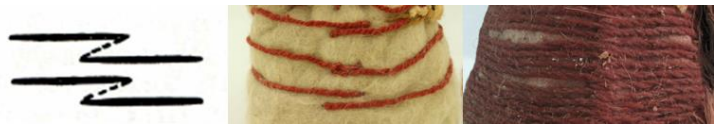

Fig. 8. Two different stem stitches.

Evidence shows that two different types of stemstitches can be recognized on the felt caps from SRC. One is simple; the other is much more elaborate. The simple stem-stitch refers to the method to embroider several rows (2-6 rounds) of threads running around the body part of the cap. The interspaces between the threads are big enough to see the felt background. Contrary to the simple ones, the elaborate ones are more complex and there are always tens of threads running on the caps from bottom to the top, covering the entire body part of the cap. The excavator from XIA suggests that the simple string trimmings always found on the caps which belong to female deceased, while the elaborate ones appear on the male's headgear. The gender difference of the string trimmings on the felt caps from SRC may indicate a primitive gender identification of those ancient people.

Blanket-stitches are another stitch which were also widely used in the thread ornaments on the felt caps from this cemetery. It always appears at the bottom edge of the felt caps. The height of the stitch varies from $0.5-2.5 \mathrm{~cm}$ while the width from $1-3 \mathrm{~cm}$. The function of this stitch was not only keeping the edge of felt cap clean and neat, but also decorating the cap in a specific way. According to the unearthed felt caps, it seems this kind of blanket-stitches always appear along with the elaborate stem-stitches on the felt caps of male mummies. (See "Fig. 9")

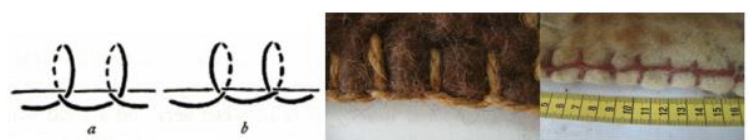

Fig. 9. Blanket-stitches.

\section{B. Feathers}

Feathers were another common decorative material for the ancient people. Archaeological evidence shows that feather ornaments were always inserted on the left sides of the felt caps from SRC. The fluffy feathers in different shades of yellow and brown look very pretty against the dim background of felt.

There are two different types of feather ornaments on the caps. One is called plume, and the other one called panache [7]. Plume refers to a kind of single feather ornament, which made by tying a tuft of soft feather (presumably feathers from the ventral parts of birds) to a wooden peg with yarns or animal sinews. The point of the peg is sharp enough to insert the plume to the felt cap. And this kind of feather ornaments often appears in pairs on the caps of female deceased. On the contrary, panache refers to feather ornaments which made by tying a group of plumes together. The making process of panache should contain two steps: first, make a single plume by tying a thick feather (presumably feathers from the wings or tails of birds) to a wooden peg with sinews or yarns. Second, fasten several (usually 4-5) single plumes together to a small transverse peg to form a feather fun. Each shaft has a sharp end so that the panache made by plumes could be easily inserted into the felt hats. Evidence showed that this kind of feather trimmings always found on the hat of male deceased.

From the material to handicrafts, the whole process of making feather decoration gives us obvious suggestion that ancient people from SR always kept a common understanding of gender. It is easy to connect the single soft plumes to the female fertility, which might be seen as the most important female quality pursued by the ancient ancestors. Meanwhile, the panaches made by the thick feathers on men's hats may symbolize the strong power of the males. (See "Fig. 10")

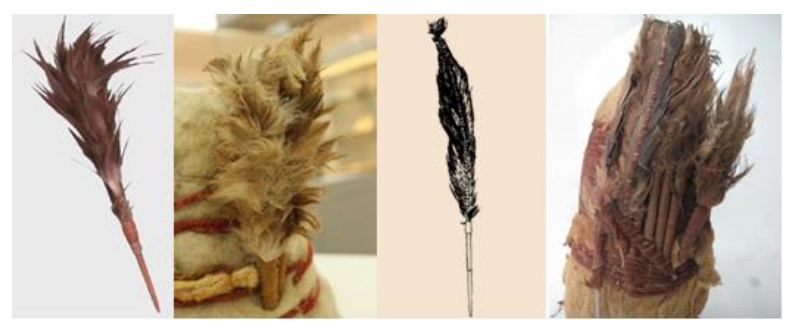

Fig. 10. Plumes and panaches.

\section{Weasel pelts}

Apart from feathers, weasel pelts (or ermine skin) were another favorite elements utilized by the SR people to decorate their headgear. It seems as if the weasel pelts combined with the string trimmings adorned most of the felt caps from SRC. Some caps have 2 weasels, one for left while the other for right, tied together in front of the caps. Others are with one 
weasel skin split into 2 parts, and only the head of the weasel was complete and fastened in front of the caps. Few of them were fastened on the caps as a whole. They were fastened by threads at heads, abdomens and feet. The length of the weasels is around $21-28 \mathrm{~cm}$, and the split weasels just matches the length of the string trimmings on the body part of the caps. As regards the motivation of the feather ornaments, Victor. H. Mair suggests that the weasel pelts and feathers might also have symbolized hunting prowess aside from their ornamental values [8]. (See "Fig. 11")

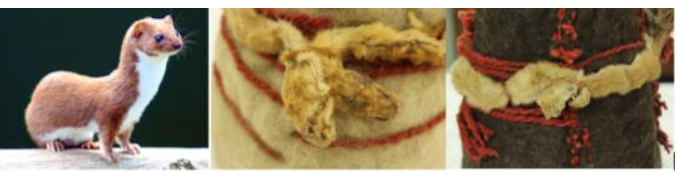

Fig. 11. Weasel pelts on the felt caps.

\section{DISCUSSION}

The SRC is one of the most important archaeological sites in the Bronze Age Xinjiang, China. And the felt caps unearthed from this cemetery is one of the most characteristic burial items which reflect the special cultural customs of ancient dwellers of Tarim Basin. It is attested that felt caps from SRC is not the only case. There are also some other similar headgear found from other place in the same period of Bronze Age Xinjiang. The felt caps from The Keriya Northern Tomb which is 595 kilometers west from the SRC have confirmed this fact. In addition, felt caps from the LS, LT, LC, LF (excavated by Stein, 1913-1916), Grave 36 (G36, excavated by Hedin, 1934), and Qäwrighul (Chinese: Gumugou, excavated by Wang Binghua, 1979) in Lop-nor region give us further evidence of it. According to the archaeological finds, the straight edge felt caps are the typical style in both SRC and KNT (Keriya Northern Tomb), while the hood-like felt caps with earflaps are much more common in other sites of Bronze Age Xinjiang and are carried on by the later Bronze Age people in Wupu (1200BC) near Qumul (Chinese: Hami). (See "Fig. 12")

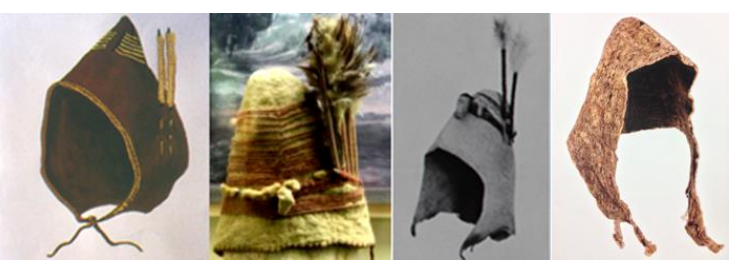

Fig. 12. Felt caps found in other Bronze Age sites of Xinjiang (felt caps from Gumugou, KNT, G 36 and Wupu) [9].

For comparison, some other Bronze Age sites outside China should be noted. For example the pointed crown caps was found in the sculptures from Hittite of ancient Anatolia around 1600 BC. And images from Assyrian relief sculptures in ancient Mesopotamia which date back to $900-800 \mathrm{BC}$ furthered the evidence, and both straight-edge cap and hood-like cap were found in those sculptures. The hats wearing by the Scythian tribute bearers in the famous bas-relief from Darius the Great's audience hall at Persepolis (515 BCE) in ancient Persian prove that Scythian also wearing similar hood-like caps. And images on Scythian vessel (400BC-350BC) discovered from south Russian steppes give us some new clues about this. Those similar caps found in other Bronze to Iron Age sites may indicate some possible links between SRC mummies and early dwellers from other place of ancient Eurasia, but more evidence are still needed to attest any hypothesis about it. (See "Fig. 13")

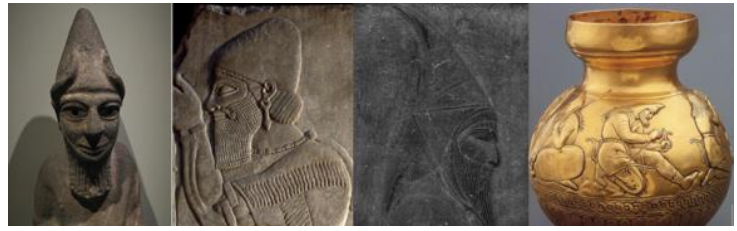

Fig. 13. The caps from Hittite, Assyrian, Persepolis and Russian [10].

As regards the cultural significance of those felt caps found in SRC, one thing has to be pointed out. It seems that most of the felt caps are new and had not been used before the funeral. The same case happened with the leather boots. Therefore, we presumed that those caps were just prepared for the burial ritual. It is hard to say whether those people wearing this style of caps in their daily lives or not, but one thing for sure is that those felt caps found in SRC are with special importance for the SR natives. As Karlene Jones Bley suggested, the ancient tall hats are the intermedia for people to build their connections with gods [11]. Some of the felt caps from SRC are very tall (more than 30 $\mathrm{cm}$ ), and the red strings, feathers and weasel pelts decorated on the SR caps might also be a proof of that.

Another interesting thing should be noted is the gender characteristic of the ornaments on the caps. As already mentioned in the ornaments section, there are obvious differences between male and female caps, which remind us about other items found in SRC with gender distinctions. For example, the tassels of the woolen cloaks are on the top part for women and bottom part for men; the loincloths are belt-like for males and skirt-like for females; the poplar posts in front of the male tombs are oar-shaped, while female are torpedoes-shaped, etc. The combs and wooden phalluses in women's coffin and feather arrows \& mortuary bows in men's give us further evidence of the mysterious gender culture of SRC. It is believed that the emphasis of gender difference indicates strong procreative worship of those ancient people, and all the burial goods shows the same wish for great reproductive capacity. 


\section{CONCLUSION}

These observations about felt caps unearthed from SRC may certainly contribute to enlarge our knowledge about the Bronze to Iron Age dwellers of Tarim Basin, although some other important aspects of ancient headgear and cultural interrelations in Xinjiang, China need to be explored by future research. Meanwhile, we would like to thank Zhao Feng, Chief curator of China National Silk Museum, and Li Wenying, Director of Xinjiang Institute of Archaeology, for give us this great opportunity to research those cultural relics from SRC. And we also thank all the colleagues who have been involved in this project.

\section{References}

[1] Vivi Sylwan. Woollen Textiles of the Lou-Lan People. Stockholm, 1941. pp.45-54; Folke Bergman.Archaeological researches in Sinkiang Especially the Lop-Nor Region. Stockholm,1939. (XinjiangKaoguji, translated by Wang Anhong, 1997, Urumqi, pp88-89.)

[2] Willow Mullins. 2009. Felt (Textile that Change the World). Oxford. pp1.

[3] Barber, E.J.W. Prehistoric Textiles: The Development of Cloth in the Neolithic and Bronze Ages. Princeton. 1991.pp217.

[4] Barber, E.J.W. The Mummies of Ürümchi. New York. 1999.pp76.

[5] Zhang Dake. New Notes on Historical Records(Shiji Quanben Xinzhu). Xi'an. 1990.pp1-8.

[6] Vivi Sylwan. Woollen Textiles of the Lou-Lan People. Stockholm, 1941. pp. 51.Fig.12.

[7] The terms "plumes" and "panache" is according to Vivi Sylwan. 1941.pp38-39.

[8] Mair, V H. The Rediscovery and Complete Excavation of Ordek's Necropolis. Journal of Indo European Studies. 2006. 34(3/4), pp296.

[9] Li Xiaobing. Study on Ethnic Costumes of West Region in China (Zhongguo Xiyu Minzu Fushi Yanjiu). Urumqi. 1995, pp66; Vivi Sylwan, Woolen Textiles of the Lou-Lan People, 1941, PLATE VII.1; Hami Museum, Marrow of Culture Relics in Hami, 2013, pp122.

[10] See Pictures from http://www.clevelandart.org/art/1971.45; http://www.britishmuseum.org/;http://www.pinterest.com/; http://hermitagemuseum.org/

[11] Karlene Jones Bley. (Ding lanlan translated). Tall Hats: Reaching to the Sky (Gaomao, Zhitong Tianguo). Steppe Cultural Relics (Caoyuan Wenwu). 2012(1). pp110-114. 